Original Article

\title{
Effects of spaced retrieval training with errorless learning in the rehabilitation of patients with dementia
}

\author{
Jong SiK JAng, MS ${ }^{1)}$, JAe Shin Lee, PhD, $\mathrm{OT}^{2)}$, Doo Han Yoo, PhD, OT ${ }^{2 *}$ \\ 1) Department of Occupational Therapy, Far East University, Republic of Korea \\ 2) Department of Occupational Therapy, Konyang University: 158 Gwanjeodong-ro, Seo-gu, Daejeon \\ 302-832, Republic of Korea
}

\begin{abstract}
Purpose] Among the non-pharmacological interventions for dementia, spaced retrieval training (SRT) is a good method for rehabilitating cognition. The purpose of this study was to examine effects of SRT with errorless learning (EL) in the rehabilitation of patients with dementia. [Subjects and Methods] Twenty-nine participants with vascular dementia (VD) and Alzheimer's disease (AD) participated in the present study. The Korean version of the Consortium to Establish a Registry for Alzheimer's disease (CERAD-K) and Modified Barthel Index (MBI) were performed to assess the changes in the neuropsychological performance and the independent activities of daily living after SRT with EL. All tests were administered both before and after SRT with EL. Each SRT with EL intervention was performed for 30 minutes per day for 5 weeks. SPSS for Windows version 18.0 was used for statistical analysis. [Results] All items of the CERAD-K score of the VD group except for constructional praxis increased significantly after the SRT with EL intervention, but no significant differences from the AD group were found. The Korean version of the geriatric depression scale (GDS-K) of the VD group increased significantly after the SRT with EL intervention. The mean MBI scores of each group showed no significant difference after the intervention. [Conclusion] SRT with EL is an effective intervention for memory training of patients with dementia. Future research using sufficient sample sizes will be needed to obtain strong evidence for comparing not only the before and after intervention data but also between the groups.

Key words: Dementia, Errorless learning, Spaced retrieval training
\end{abstract}

(This article was submitted Apr. 23, 2015, and was accepted May 25, 2015)

\section{INTRODUCTION}

With the increasing elderly population, dementia has become one of the most common social problems ${ }^{1)}$. Because memory impairment is the core symptom of dementia, the majority of early interventions aim to slow cognitive decline. In recent years, pharmacological interventions have commonly been used to achieve this ${ }^{2}$. However, studies into their use for dementia patients have found them to have limited effectiveness ${ }^{3}$. Recent studies, however, have focused on non-pharmacological cognitive interventions to alleviate the cognitive deficits related to dementia ${ }^{4,5)}$

Non-pharmacological cognitive interventions for dementia include re-learning strategies that promote the management ability to reduce cognitive impairment ${ }^{3,6)}$. Cognitive interventions differ from pharmacological interventions in that they consider the interaction between the patients and

*Corresponding author. Doo Han Yoo (E-mail: glovia@ hanmail.net)

(C2015 The Society of Physical Therapy Science. Published by IPEC Inc. This is an open-access article distributed under the terms of the Creative Commons Attribution Non-Commercial No Derivatives (by-ncnd) License $<$ http://creativecommons.org/licenses/by-nc-nd/3.0/>. therapist, as well as the needs of the patient ${ }^{7)}$. Cognitive interventions have been shown to temporarily slow the rate of cognitive decline and the loss of functional abilities. On the other hand, they can fail because cognitive rehabilitation interventions that are beneficial for the patients with dementia require significant effort and attention.

Spaced retrieval training (SRT) is a good candidate strategy for cognitive intervention in dementia because it does not require considerable cognitive effort ${ }^{8)}$. SRT is an expanded interval timer with independent data-tracking and timing of up to 3 targets $^{4}$. SRT recalling targets over increasingly longer periods of time has been proven to result in improved learning in people with mild to moderate dementia. This training regime was developed based on the expanding rehearsal technique which was originally developed for improving memory in cognitively intact persons ${ }^{9)}$. Errorless learning (EL) is a teaching technique that prevents the making of mistakes while subjects are learning a new skill or acquiring new information, and it is conducted together with SRT or the vanishing cues technique. SRT with EL is a technique for training the memory which uses an expanded interval that increases with correct responses and decreases with incorrect ones ${ }^{10)}$.

Kinsella et al. ${ }^{11)}$ suggested that SRT would be beneficial for individuals with dementia because it utilizes the rela- 
tively unimpaired automatic implicit memory system. SRT can be used with EL because it minimizes the possibility of error during the acquisition phase of learning by ensuring mistakes. In addition, SRT has a spacing effect that is retrieved more effectively when trials are distributed over time. SRT appears to be attracting attention within the field of dementia treatment because of these advantages ${ }^{12,13)}$.

Non-pharmacological interventions for dementia, such as music, art, physical activity, and gardening, are often performed, but cognitive intervention of SRT with EL has rarely been conducted in clinical settings. In particular, studies aimed at observing the effects of SRT in a clinical environment are difficult to perform. Therefore, the purpose of this study was to examine effects of SRT with errorless learning (EL) for the rehabilitation of patients with dementia.

\section{SUBJECTS AND METHODS}

Twenty-nine participants with vascular dementia (VD) and Alzheimer's disease (AD) participated in the present study. The study participants were enrolled from among inpatients in a rehabilitation-care hospital of Korea. All the subjects provided their written informed consent to participation in the experiment in accordance with the ethical principles of the Declaration of Helsinki. Subjects diagnosed with dementia including major depressive disorder by the DSM-IV criteria and those with serious medical and neurological problems that could have affected their mental function were excluded.

Table 1 lists the general characteristics of the 29 participants. The AD group had a higher average age than the VD group, and had lower mean values in the Korean version of Mini-Mental Status Evaluation (MMSE-KC), and the Korean version of geriatric depression scale (GDS-K), as well as lower education levels than the VD group.

MMSE-KC was used to provide an index of the current cognitive status ${ }^{14)}$. The maximum score on MMSE-KC is 30. On this measure, healthy participants' scores range from 27 to 30 . Scores of less than 26 mean that subjects have some cognitive problems. GDS-K was administered to obtain a measure of the affective status ${ }^{15}$ ). This measure consists of 30 items with each item scoring 1 point, and higher total scores indicate a higher level of depression. The cognitive functions were investigated using the Korean version of the Consortium to Establish a Registry for the Alzheimer's disease (CERAD-K) battery, which consists of 8 subtests $^{16)}$. The independence of activities of daily living (ADL) was examined using the modified Barthel Index which consists of 10 subtests.

The SRT with EL was administered by first providing the patients with a prompt question and associated target response. When successful at recalling the response, the patient was required to recall the target information at longer inter-trial intervals. If at any time the patient was unable to recall the response, they were provided with the information and asked to repeat it. The training was considered successful if the patient correctly responded to the prompt question at the beginning of each session $(45,90,180,360$, and 720 sec.). The set of words consisted of one to five unrelated imagery words. All words were composed of 100 different
Table 1. General characteristics of the subjects $(n=29)$

\begin{tabular}{lcc}
\hline Characteristics & $\mathrm{VD}(\mathrm{n}=22)$ & $\mathrm{AD}(\mathrm{n}=7)$ \\
\hline Gender (male/female) & $13 / 9$ & $2 / 5$ \\
Age (years) & $58.9 \pm 9.9$ & $78.1 \pm 4.4$ \\
Education (years) & $7.9 \pm 5.0$ & $1.7 \pm 2.9$ \\
MMSE-KC & $15.7 \pm 5.1$ & $9.2 \pm 3.2$ \\
GDS-K & $18.8 \pm 5.6$ & $15.5 \pm 3.4$ \\
\hline
\end{tabular}

VD: vascular dementia; AD: Alzheimer dementia; MMSE$\mathrm{KC}$ : Korean version of mini mental status evaluation in the CERAD-K assessment packet, GDS-K: Korean version of geriatric depression scale

words presented as high frequency words by the National Institute of the Korean Language. If a subject repeatedly failed to remember a word for 45 seconds, the subject was excluded. If the subject remembered the word in two sessions $(720 \mathrm{sec} . \times 2$ session), the number of words added ranged from one to five. During each interval, the subject was asked to perform inter-retrieval activities in order to prevent them from rehearsing the given set of words. The interretrieval activities consisted of simple physical activities that did not require much memory function (e.g., jenga, puzzle, pegboards). SRT with EL interventions were performed for 30 minutes per day for 5 weeks.

SPSS for Windows version 18.0 was used for the statistical analysis, and p values less than 0.05 were considered significant. Wilcoxon's matched-pairs signed-rank test was used to compare the pre- and post-test neuropsychological, depression, and ADL performances of each group.

\section{RESULTS}

As listed in Table 2, all items of the CERAD-K score in the VD group except for constructional praxis increased significantly after the SRT with EL intervention, but their values were similar to those of the AD group. Significant differences were observed in the gain of the verbal fluency test, the Boston naming test, MMSE-KC, word list memory, word list recall, word list recognition, and constructional recall in the VD group between before and after the intervention. There were no significant differences in the subtests in the $\mathrm{AD}$ group between before and after the intervention.

Table 3 compares the effects of the SRT on GDS-K and MBI of the dementia groups. GDS-K of VD increased significantly after the SRT with EL intervention. The mean MBI score of each group was similar after the intervention.

\section{DISCUSSION}

The efficacy of SRT with EL was examined using 29 patients with dementia. The subjects with dementia could perform the SRT with EL. In particular, the SRT with EL intervention was beneficial for the VD patients. This suggests that the cognitive ability of dementia patients may be improved by SRT with EL.

Previous studies have shown that dementia patients have decreased communication skills and voluntary activity because of their reduced cognitive function. Therefore, it 
Table 2. Comparison of the effect of SRT on CERAD-K of dementia patients

\begin{tabular}{lcccc}
\hline & \multicolumn{2}{c}{ VD group $(\mathrm{n}=22)$} & \multicolumn{2}{c}{ AD group $(\mathrm{n}=7)$} \\
\hline Verbal fluency test & $5.82 \pm 2.74$ & $7.18 \pm 3.13^{*}$ & $3.29 \pm 1.60$ & $4.29 \pm 2.56$ \\
Boston naming test & $7.41 \pm 3.20$ & $8.14 \pm 3.11^{* *}$ & $3.71 \pm 2.14$ & $3.57 \pm 2.30$ \\
MMSE-KC & $15.73 \pm 5.17$ & $17.27 \pm 4.65^{* *}$ & $9.29 \pm 3.25$ & $10.00 \pm 4.32$ \\
Word list memory & $8.82 \pm 3.84$ & $10.77 \pm 4.46^{*}$ & $2.57 \pm 3.55$ & $2.86 \pm 3.80$ \\
Constructional praxis & $6.00 \pm 2.91$ & $6.14 \pm 3.31$ & $5.71 \pm 1.98$ & $5.71 \pm 2.06$ \\
Word list recall & $2.45 \pm 1.74$ & $3.36 \pm 1.87^{* *}$ & $0.86 \pm 1.57$ & $0.86 \pm 1.57$ \\
Word list recognition & $4.36 \pm 3.03$ & $5.68 \pm 3.47^{*}$ & $1.86 \pm 1.95$ & $1.29 \pm 2.21$ \\
Constructional recall & $2.41 \pm 2.52$ & $3.64 \pm 2.36^{* *}$ & $0.43 \pm 1.13$ & $0.43 \pm 1.13$ \\
\hline
\end{tabular}

SRT: spaced retrieval training; CERAD-K: Korean version of consortium to establish a registry of Alzheimer's disease; VD: vascular dementia; AD: Alzheimer dementia; Values are expressed as mean \pm SD Significant difference from pre-test, ${ }^{*} \mathrm{p}<0.05,{ }^{* *} \mathrm{p}<0.01$

Table 3. Comparison of the changes in GDS-K and MBI between before and after the intervention

\begin{tabular}{lcccc}
\hline & \multicolumn{2}{c}{ VD group $(\mathrm{n}=22)$} & \multicolumn{2}{c}{ AD group $(\mathrm{n}=7)$} \\
\hline & Before & After & Before & After \\
\hline GDS-K & $18.8 \pm 5.6$ & $17.2 \pm 6.1^{*}$ & $15.5 \pm 3.4$ & $17.8 \pm 4.5$ \\
MBI & $57.2 \pm 16.5$ & $57.3 \pm 16.7$ & $91.2 \pm 4.1$ & $90.8 \pm 4.0$ \\
\hline
\end{tabular}

GDS-K: Korean version of the geriatric depression scale; MBI: Modified Barthel index; Values are expressed as mean \pm SD Significant difference from pre-test, $* \mathrm{p}<0.05$

is more difficult for dementia patients to have interpersonal relationships and engage in social participation ${ }^{17}$. Cognitive interventions are essential to maintain the motivation for community participation. In addition, they are required for enhancing memory in cognitive interventions. Considerable research has focused on the efficacy of cognitive intervention in rehabilitation. SRT as a cognitive approach has been reported to be effective ${ }^{18)}$. In addition, SRT consistently resulted in beneficial outcomes in the present study.

The in-session maximum retention span was maintained at 720 seconds during SRT for the VD and AD groups. In addition, the intervention time was set at 30 minutes, considering the clinical environment and the disease characteristics. SRT was previously administered in an in-session retention span of 1,440 seconds for mild dementia, but 720 seconds was used in the present study because of the various characteristics of dementia.

SRT with EL had a significant effect on the performance of the VD group in the CERAD-K except for the item of constructional praxis. After the SRT with EL, the VD group showed superior cognitive performance compared to the post-test values of the AD group. Findings similar to those of the VD group have been reported by previous studies ${ }^{19)}$. Although not statistically significant, SRT with EL as a cognitive approach elicited improvements in the $\mathrm{AD}$ group. Moreover, increasing the number of target words during the SRT intervention may enhance the memory function in the early stages of $\mathrm{AD}$, and might improve the acquisition of new information by patients with dementia ${ }^{20)}$.

No study has reported significant improvements in ADLs after SRT with EL ${ }^{18)}$. The present study also found no significant improvement in ADLs. It is our opinion that this is due to the difficulty in accurately calculating individual effect sizes for the ADLs. MBI has limited ability to compare the changes in the performance of patients with dementia after a short training period.

A limitation of the present study is that the changes were not compared with another dementia group. While there were before and after changes in the VD and $\mathrm{AD}$ groups, it was difficult to identify differences between the groups. Therefore, additional research will be needed to determine the effects of SRT on AD patients according to the method of intervention. Future research using sufficient sample sizes will be needed to obtain strong evidence for its efficacy not only in pre- and post-test results, but also between groups.

\section{REFERENCES}

1) Dramé M, Lang PO, Jolly D, et al.: Nursing home admission in elderly subjects with dementia: predictive factors and future challenges. J Am Med Dir Assoc, 2012, 13: 83.e17-83.e20. [Medline] [CrossRef]

2) Aderinwale OG, Ernst HW, Mousa SA: Current therapies and new strategies for the management of Alzheimer's disease. Am J Alzheimers Dis Other Demen, 2010, 25: 414-424. [Medline] [CrossRef]

3) Fitzsimmons $\mathrm{S}$, Barba B, Stump M, et al.: Nonpharmacological interventions in long-term care: feasibility and recent trends. J Gerontol Nurs, 2014, 40: 10-14. [Medline] [CrossRef]

4) Creighton AS, van der Ploeg ES, O'Connor DW: A literature review of spaced-retrieval interventions: a direct memory intervention for people with dementia. Int Psychogeriatr, 2013, 25: 1743-1763. [Medline] [CrossRef]

5) Erkes J, Raffard S, Meulemans T: [Spaced-retrieval in patients with Alzheimer's disease. Critical review and clinical applications]. Psychol Neuropsychiatr Vieil, 2009, 7: 275-286. [Medline]

6) Kim S: Cognitive rehabilitation for elderly people with early-stage Alzheimer's disease. J Phys Ther Sci, 2015, 27: 543-546. [Medline] [CrossRef]

7) Baune BT, Renger L: Pharmacological and non-pharmacological interventions to improve cognitive dysfunction and functional ability in clinical depression-a systematic review. Psychiatry Res, 2014, 219: 25-50. [Medline] [CrossRef]

8) Abrahams JP, Camp CJ: Maintenance and generalization of object naming training in anomia associated with degenerative dementia. Clin Gerontol, 1993, 12: 57-72. [CrossRef]

9) Han JW, Oh K, Yoo S, et al.: Development of the ubiquitous spaced retrieval-based memory advancement and rehabilitation training program. Psychiatry Investig, 2014, 11: 52-58. [Medline] [CrossRef]

10) Hopper T, Bourgeois M, Pimentel J, et al.: An evidence-based systematic 
review on cognitive interventions for individuals with dementia. Am J Speech Lang Pathol, 2013, 22: 126-145. [Medline] [CrossRef]

11) Kinsella GJ, Ong B, Storey E, et al.: Elaborated spaced-retrieval and prospective memory in mild Alzheimer's disease. Neuropsychol Rehabil, 2007, 17: 688-706. [Medline] [CrossRef]

12) Thivierge S, Simard M, Jean L, et al.: Errorless learning and spaced retrieval techniques to relearn instrumental activities of daily living in mild Alzheimer's disease: A case report study. Neuropsychiatr Dis Treat, 2008, 4: 987-999. [Medline]

13) Crowe J, Linda G: Errorless learning and spaced retrieval training for clients with Alzheimer's dementia. Phys Occup Ther Geriatr, 2013, 31 254-267. [CrossRef]

14) Yoo DH, Hong DG, Lee JS: The standardization of the clock drawing tes (CDT) for people with stroke using Rasch analysis. J Phys Ther Sci, 2013 , 25: 1587-1590. [Medline] [CrossRef]

15) Bae JN, Cho MJ: Development of the Korean version of the Geriatric Depression Scale and its short form among elderly psychiatric patients. J Psy- chosom Res, 2004, 57: 297-305. [Medline] [CrossRef]

16) Lee JH, Lee KU, Lee DY, et al.: Development of the Korean version of the Consortium to Establish a Registry for Alzheimer's Disease Assessment Packet (CERAD-K): clinical and neuropsychological assessment batteries. J Gerontol B Psychol Sci Soc Sci, 2002, 57: 47-53. [Medline] [CrossRef]

17) Fratiglioni L, Wang HX, Ericsson K, et al.: Influence of social network on occurrence of dementia: a community-based longitudinal study. Lancet, 2000, 355: 1315-1319. [Medline] [CrossRef]

18) Grandmaison E, Simard M: A critical review of memory stimulation programs in Alzheimer's disease. J Neuropsychiatry Clin Neurosci, 2003, 15: 130-144. [Medline] [CrossRef]

19) Lee SB, Park CS, Jeong JW, et al.: Effects of spaced retrieval training (SRT) on cognitive function in Alzheimer's disease (AD) patients. Arch Gerontol Geriatr, 2009, 49: 289-293. [Medline] [CrossRef]

20) Germano C, Kinsella GJ: Working memory and learning in early Alzheimer's disease. Neuropsychol Rev, 2005, 15: 1-10. [Medline] [CrossRef] 